\title{
The safety of transcranial magnetic stimulation with deep brain stimulation instruments.
}

\author{
Yoshio Shimojima $^{\text {ab }}$, Hiroshi Morita ${ }^{\text {a*, Noriko Nishikawa }}{ }^{\text {ac }}$, Minori Kodaira ${ }^{a}$, Takao \\ Hashimoto $^{\text {ad }}$, and Shu-ichi Ikeda ${ }^{a}$.
}

${ }^{a}$ Department of Medicine (Neurology) Shinshu University School of Medicine

Asahi 3-1-1 Matsumoto 390-8621, Japan

Present address:

${ }^{\mathrm{b}}$ Department of Neurology, Iida Municipal Hospital, Iida Japan.

${ }^{c}$ Department of Clinical Pharmacology and Therapeutics, Ehime University School of Medicine, Ehime Japan.

${ }^{\mathrm{d}}$ Center for Neurological Diseases, Aizawa Hospital, Matsumoto Japan.

Address correspondence to: Dr. Hiroshi Morita.

Department of Medicine (Neurology)

Shinshu University School of Medicine

Asahi 3-1-1 Matsumoto 390-8621, Japan

Fax +81-263-37-3427

hmorita@shinshu-u.ac.jp 


\begin{abstract}
Objectives: Transcranial magnetic stimulation (TMS) has been employed in patients with an implanted deep brain stimulation (DBS) device. We investigated the safety of TMS using simulation models with an implanted DBS device. Methods: The DBS lead was inserted into plastic phantoms filled with dilute gelatin showing impedance similar to that of human brain. TMS was performed with three different types of magnetic coil. During TMS (1) electrode movement, (2) temperature change around the lead, and (3) TMS-induced current in various situations were observed. The amplitude and area of each evoked current were measured to calculate charge density of the evoked current. Results: There was no movement or temperature increase during $0.2 \mathrm{~Hz}$ repetitive TMS with $100 \%$ stimulus intensity for 1 hour. The size of evoked current linearly increased with TMS intensity. The maximum charge density exceeded the safety limit of $30 \mu \mathrm{C} / \mathrm{cm}^{2} /$ phase during stimulation above the loops of the lead with intensity over $50 \%$ using a figure-eight coil. Conclusions: Strong TMS on the looped DBS leads should not be administered to avoid electrical tissue injury. Subcutaneous lead position should be pay enough attention for forthcoming situations during surgery.
\end{abstract}

\title{
Key words:
}

Deep Brain Stimulation (DBS), Transcranial Magnetic Stimulation (TMS), safety, tissue injury, Motor Evoked Potential (MEP) 


\section{Introduction}

In transcranial magnetic stimulation (TMS), a magnetic field is generated around the coil by the electric current discharged from the large volumetric condensers. Such a strong magnetic pulse can break precision electrical instruments such as deep brain stimulation (DBS) generator when the coil is placed over such devices [1]. The magnetic field around the coil induces electric current in brain tissue and causes excitation of neuronal cells. TMS has the advantage of being less painful and uncomfortable than electric stimulation, and it is possible to convey impulses toward the deep brain tissues without attenuation of the stimulus intensity [2,3]. This is useful not only for quantitative estimation of pyramidal tract function but also as a tool to investigate functions of the central nervous system, such as the basal ganglia-cortex interaction. TMS has been used to estimate pathophysiological mechanisms of symptoms in Parkinson's disease (PD) [4]. On the other hand, DBS is one of the most useful methods to treat extrapyramidal disorders such as PD [5], and the number of patients with DBS devices implanted is increasing. The combination of DBS and TMS in PD is expected to provide information regarding the physiological mechanisms of extrapyramidal disorders. Some studies using TMS in DBS implanted patients have been reported without any serious complication or damage to the DBS system [6-10]. The device manufacturer, however, strictly indicates that TMS should not be administered to patients with an implanted DBS device due to the risk of brain damage [7]. Although the safety or usefulness of TMS in patients with a DBS system implanted has been reported [1,6-10], more systematic investigations are necessary before application of TMS to patients with an implanted DBS system becomes widespread. In this study, we systematically investigated the safety of TMS in patients with an implanted DBS device using a phantom simulation, and propose safety guidelines for the use of TMS. 


\section{Methods}

\section{Instruments and tools}

TMS was performed with Magstim 200 stimulator (The Magstim Co., Ltd, Dyfed, UK) and several kinds of TMS coil (round coil, figure-eight coil and double cone coil). DBS system was composed of a generator (Soletra ${ }^{\mathrm{TM}}$ Model, Medtronic, Minneapolis, MN, USA) and an electrode with four tip (model 3389, Medtronic, Minneapolis, MN, USA). We prepared two phantoms, one was a small box $(5.5 \times 10 \times 20 \mathrm{~cm}$, made of acrylic resin $)$ and the other was a head phantom composed of a hollow plastic model (specially made: circumference of the head $56 \mathrm{~cm}$, major axis $21 \mathrm{~cm}$, minor axis $16 \mathrm{~cm}$, inner volume $4500 \mathrm{ml}$, thickness of the plastic skull 3.0mm). The small box was used for simulation for lead movement and increase of temperature with TMS. To measure TMS induced current with TMS we used the head shaped phantom. We filled both phantoms with some dilute gelatin with the almost same consistency as brain tissue. To achieve the same impedance as human brain (about 500 $2000 \Omega$ ), we mixed a small amount of sodium chloride into gelatin.

In the small box simulation, we made a hole on the top and inserted a DBS electrode into the centre of the box. In the head phantom, we made a small hole in the left frontal position and inserted a DBS lead through the hole directed toward the hypothetical subthalamic nucleus, as we do for an actual stereotactic operation (Figure 1). A grounded copper plate electrode $\left(50 \mathrm{~cm}^{2}\right)$ was inserted into the inferior portion of the head model. We attached a pouch filled with chilled gel on the back of the coil to prevent overheating of the coil during continuous stimulation for 1 hour.

\section{Can TMS cause movement of the DBS lead in the brain?}

The DBS lead was inserted through a hole on the top of the box that was or was not filled with gelatin, and was fixed to the box at a point $10 \mathrm{~cm}$ from the end of the lead with a plastic cap, a device used for human DBS surgery. TMS was administered using the figure-eight 
coil just above the insertion point of the lead. During stimulation, we recorded images of the electrode with a commercial digital video camera to monitor movement of the electrode with TMS. Stimulus intensity used was $100 \%$.

\section{Can TMS overheat the DBS lead?}

A DBS lead and a thermometer were inserted into the box filled with gelatin. We measured the temperature of gelatin surrounding a lead while TMS was administered with the figure-eight coil over the insertion point. Stimulation was performed with $100 \%$ stimulus intensity of maximum stimulator output at a frequency of $0.2 \mathrm{~Hz}$ for one hour.

\section{How much current evoked by TMS does spread to the brain through implanted electrode?}

The DBS lead inserted into the head phantom filled with gelatin was used for this observation. Electrical brain damage depends on induced charge density rather than on the voltage of induced current. The charge density is a function of voltage, current duration, impedance of electrodes, and size of electrodes. Therefore, charge density must be calculated from amplitude and duration of induced current, and impedance. Impedance is also the function of current size; therefore, we must determine the relationship between impedance and induced current.

To calculate these relationships, we first systematically measured impedance between electrode terminals in the phantom with variable currents using generator devices. Another side of insulated DBS electrode was connected to the DBS generator (Soletra ${ }^{\mathrm{TM}}$, Medtronic, Minneapolis, MN, USA). Duration of current and pairs of electrode terminals were also systematically changed and measured impedance in each situations using programmer (7432 programmer for Soletra ${ }^{\mathrm{TM}}$, Medtronic, Minneapolis, MN, USA). With these measurements, we could develop a numerical formula for the relationship between induced current strength (amplitude and duration) and impedance of this system.

Thereafter, two of four electrodes on the generator side were connected to an 
oscilloscope and a recording system (POWER 1401 and Signal2 software, Cambridge Electric Design, Cambridge, UK) to record current evoked by TMS.

Although charge density (microcoulombs $(\mu \mathrm{C}) / \mathrm{cm}^{2} /$ phase) was defined as [Voltage (volt) $\times$ duration $(\mu \mathrm{s}) /$ impedance $(\Omega) / 0.06\left(\mathrm{~cm}^{2}\right.$, electrode area)], TMS-induced current was not rectangular. We, therefore, measured the product of current intensity by duration as the area of induced current, and finally estimated the approximate charge density from measured size of current and calculated impedance using a numerical formula developed from the above measurement.

\section{3-1. Relationship between stimulus strength and induced current}

The DBS lead was laid on the surface of the simulation head from the insertion point at the left frontal position to the neck via the back of the ear without making any loop. TMS was administered with a round coil over the $\mathrm{Cz}$ point and insertion point, and with a figure-eight coil over the estimated position of the ipsilateral primary motor cortex and insertion point, and with a double cone coil over the Cz point. Stimulus intensity of TMS was systematically increased from 5 to $100 \%$ under each experimental setting.

\section{3-2. Positional relation between TMS coil and DBS lead and induced current}

To observe the relationship between coil positions and the strength of induced current, we systematically moved the figure-eight coil with fixed stimulus intensity over the surface of the head phantom. For systematic stimulation, we plotted the coordinate axes of lateral and vertical direction and marked lattice points every $1 \mathrm{~cm}$ on the phantom head. We temporarily defined the insertion point of the lead as the datum point. The lateral axis was marked with 17 points (from $-6 \mathrm{~cm}$ to $+10 \mathrm{~cm}$ ) from left to right, and vertical axis with 16 points (from $-5 \mathrm{~cm}$ to $+10 \mathrm{~cm}$ ) from anterior to posterior. Stimulation was thus applied to the whole head phantom using a fixed stimulus intensity of $50 \%$.

A DBS lead was fixed on the surface of the head phantom in two ways; one was 
simple fixation on the head surface from the insertion point on the left frontal position to the neck via the back of the ear, and the other with double loops measuring $3 \mathrm{~cm}$ in diameter near the insertion point. The handle of the stimulus coil of TMS was kept parallel with the vertical axis.

\section{Results}

\section{Can TMS cause movement of the DBS lead in the brain?}

Under VTR monitoring, we could not detect any movement of the DBS lead in the gelatin or any space caused by lead movement that pushed aside gelatin. Duration of the induced current was so short that VTR may not have been able to capture instantaneous change in the lead position. However, there was no detectable vibration of the gelatin around the lead, and no apparent movement of the lead even when the lead was simply hung down inside the box, which was not filled with gelatin.

\section{Can TMS overheat the DBS lead?}

Temperature around the DBS lead was equal to room temperature of $25^{\circ} \mathrm{C}$ before TMS. No increase of temperature around the lead happened during continuous TMS of $0.2 \mathrm{~Hz}$ with the maximal intensity for 1 hour.

3. How much current evoked by TMS does spread to the brain through implanted electrode?

\section{3-1. Relationship between stimulus strength and induced current}

In this experiment, we fixed the residual part of insulated lead on the surface of the model without making any loop. The shape of the induced potential was triangular biphasic (Figure 2), but the size and polarity changed with the relative position between coil and lead. The amplitude of induced current depended on the type of the coil and its relative location to the lead. Regarding use of the figure-eight coil or round coil, the induced current became larger when it was positioned closer to the lead. 
There was a linear relationship between size of induced current and stimulus intensity under all of the conditions examined (Figure 2). The maximal induced current was more than $20 \mathrm{~V}$ in these situations. The calculated estimate of impedance at this maximal induced current in this model was $1162 \Omega$. The calculated charge density with this induced current was almost $20 \mu \mathrm{C} / \mathrm{cm}^{2} /$ phase. Although this was less than the safety limit, the current theoretically becomes larger if TMS was applied over the loops of the lead that were fixed on the scalp.

\section{3-2. Positional relation between TMS coil and DBS lead and induced current}

We investigated the relationship between coil position and induced current under conditions similar to those in clinical practice. The coil moved systematically, applying stimulation to the head phantom. Amplitude of the induced current became much larger when the lead was fixed in a multi-looped manner. The maximal peak-to-peak amplitude evoked by $50 \%$ stimulus intensity with a figure-eight coil was 7.7V when the lead was fixed without looping on the surface of the head phantom (Fig. 3A). The amplitude, however, increased to $34 \mathrm{~V}$ when the lead was fixed on the phantom with double loops as shown in Figure 1A (Fig. 3B). The maximal current was recorded when the coil was placed just above the DBS lead, especially over the loop of the lead.

Figure 4 shows a relationship between the calculated charge density of induced current and its relation to the coil position. The induced charge density was maximized when the loop was positioned under the coil. Although the safety limit of charge density was $30 \mu \mathrm{C} / \mathrm{cm}^{2} /$ phase [11], the charge density increased to more than $20 \mu \mathrm{C} / \mathrm{cm}^{2} /$ phase with a stimulus intensity of 50\% and double-loop fixation (Fig 4B). This indicates that the induced current should be greater than the safety limit if stimulus intensity were increased to more than $75 \%$ or the lead was fixed after making three or more loops, which is not rare in clinical practice. 


\section{Discussion}

We demonstrated that low-frequency $(0.2 \mathrm{~Hz})$ continuous TMS does not affected to the electrode position or temperature around the lead. The TMS-induced charge density, however, increased beyond the safety limit under some conditions.

\section{Dislocation and temperature increase of DBS lead with TMS}

In our experiment, magnetic stimulation over the scalp model did not induce any movement of the inserted DBS lead. Although small vibratory movements of the lead should theoretically occur during magnetic stimulation, we could not detect such movements even when the model was not filled with gelatin. The movement of a DBS lead with TMS (100\% stimulus intensity of maximum stimulator output), therefore, should be sufficiently slight that there would not be serious dislocation of the DBS lead due to TMS.

Increased temperature would also be a serious problem that could injure brain tissue. However, continuous $(0.2 \mathrm{~Hz})$ strong magnetic stimulation did not increase the DBS lead temperature. We can, therefore, conclude that magnetic stimulation (100\% stimulus intensity of Magstim, $0.2 \mathrm{~Hz}$ ) does not induce dislocation of the DBS lead or increase the tissue temperature around the DBS lead.

\section{Intensity of induced current and safety limit}

TMS induces a substantial electric current around the tip of the implanted DBS lead. We recorded the electric current and calculated the charge density. When the TMS coil was positioned over the DBS lead, which was fixed to the scalp surface after looping several times, TMS induced a strong current around the tip of the DBS lead, which damages brain tissue. A survey of studies regarding direct electrical stimulation of animal neural tissue with charge balanced biphasic waves indicated that stimulus intensity below approximately $30 \mu \mathrm{C} / \mathrm{cm}^{2} /$ phase did not damage brain tissue [12-15]. The manufacturer of the DBS system 
therefore determined the safety limit of charge density as $30 \mu \mathrm{C} / \mathrm{cm}^{2} /$ phase [11], although another study reported that subdural repetitive $(50 \mathrm{~Hz})$ electrical stimulation of $57 \mu \mathrm{C} / \mathrm{cm}^{2} /$ phase for 5 seconds did not cause histological changes in brain tissue [16].

In our simulation, the charge density increased beyond $20 \mu \mathrm{C} / \mathrm{cm}^{2} /$ phase when TMS intensity was set to $50 \%$ and the DBS lead fixed after making double loops. As shown in Figure 2, the induced current linearly increased along with TMS intensity. The intensity of induced current is also a function of the number of loops. The induced current, therefore, became stronger when the DBS lead forms loops three or more times. The impedance of our simulations was similar to that actually measured in brain tissue during surgery, but the impedance differs among areas of the brain and varies among persons in a range from about 500 to $2000 \Omega$. With lower impedance, the charge density increases under the same induced current. We, therefore, conclude that strong TMS above the loop of the DBS lead should not be performed under any experimental condition. However, TMS at a distance from the loop seems enough safe even when a strong TMS is applied.

Another factor we should consider is the charge imbalance of the induced current because the induced current is comprised of charge imbalanced biphasic waves as shown in Figure 2. A charge-imbalanced waveform injures tissue [17]. TMS, therefore, has the potential to injure neural tissue beyond the level predicted by studies of electrically induced tissue damage.

This simulated study only indicated that each single TMS discharged electric current into the brain via DBS lead. In human, there should be other additional factors in chronic situations. We are not able to exclude the possibility that repetitive biphasic TMS has another effects. Fortunately there have not been any adverse events such as burn injury of the brain reported to date in any studies using a combination of DBS and TMS in humans. 
Although the exact safety limit of charge density in chronic human situation is obscure, the TMS above DBS lead with loop at least induces strong current into human brain. To perform TMS safely in patients with implanted DBS leads, we recommend that TMS coils should not be positioned near the loop of the DBS lead. The present findings indicate that harmful charge densities can be induced by TMS in patients with implanted DBS devices; but the current findings also showed that TMS study using the commonly applied stimulus intensity can be safely performed when TMS coils are positioned at a distance from the loops of the DBS lead. 


\section{References}

[1] Kumar R, Chen R, Ashby P. Safety of transcranial magnetic stimulation in patients with implanted deep brain electrodes. Mov Disord 1999;14:157-8.

[2] Barker AT, Jalinous R, Freeston IL. Non-invasive magnetic stimulation of human motor cortex. Lancet 1985;8437:1106-7.

[3] Sekino M, Ueno S. Comparison of current distributions in electroconvulsive therapy and transcranial magnetic stimulation. J Appl Phys 2002;91:8730-2.

[4] Cantello R, Tarletti R, Civardi C. Transcranial magnetic stimulation and Parkinson's disease. Brain Res Brain Res Rev 2002;38:309-27.

[5] Limousin P, Pollak P, Benazzouz A, Hoffmann D, Le Bas JF, Broussolle E et al. Effect of parkinsonian signs and symptoms of bilateral subthalamic nucleus stimulation. Lancet 1995;8942:91-5.

[6] Kuhn AA, Meyer BU, Trottenberg T, Brandt SA, Schneider GH, Kupsch A. Modulation of motor cortex excitability by pallidal stimulation in patients with severe dystonia. Neurology 2003; 60:768-74.

[7] Molnar GF, Sailer A, Gunraj CA, Cunic DI, Lang AE, Lozano AM et al. Changes in cortical excitability with thalamic deep brain stimulation. Neurology. 2005;64:1913-9.

[8] Hidding U, Bäumer T, Siebner HR, Demiralay C, Buhmann C, Weyh T et al. MEP latency shift after implantation of deep brain stimulation systems in the subthalamic nucleus in patients with advanced Parkinson's disease. Mov Disord 2006;21:1471-6.

[9] Pötter-Nerger M, Ilic TV, Siebner HR, Deuschl G, Volkmann J. Subthalamic nucleus stimulation restores corticospinal facilitation in Parkinson's disease. Mov Disord. 2008;23:2210-5.

[10] Fraix V, Pollak P, Vercueil L, Benabid AL, Mauguière F. Effects of subthalamic nucleus stimulation on motor cortex excitability in Parkinson's disease. Clin 
Neurophysiol. 2008;119:2513-8.

[11] Medtronic. R 3387/3389 DBS TM Technical manual. Medtronic. Minneapolis 1998

[12] Agnew WF, McCreery DB, Yuen TG, Bullara LA. Effect of Prolonged Electrical Stimulation of the Central Nervous System. In: Agnew WF, McGreery DB, editors. Neural Prostheses Fundamental Studies. (Prentice Hall Biophysics and Bioengineering Series) Upper Saddle River: Prentice Hall; 1990. p225-52.

[13] Agnew WF, Yuen TG, McCreery DB. Morphologic changes after prolonged electrical stimulation of the cat's cortex at defined charge densities. Exp Neurol 1983;79:397-411.

[14] Agnew WF, Yuen TG, McCreery DB, Bullara LA. Histopathologic evaluation of prolonged intracortical electrical stimulation. Exp Neurol 1986;92:162-85.

[15] Babb TL, Soper HV, Lieb JP, Brown WJ, Ottino CA, Crandall PH. Electrophysiological studies of long-term electrical stimulation of the cerebellum in monkeys. J Neurosurg 1977;47:353-65.

[16] Gordon B, Lesser RP, Rance NE, Hart J Jr, Webber R, Uematsu S et al. Parameters for direct cortical electrical stimulation in the human: histopathologic confirmation. Electroencephalogr Clin Neurophysiol 1990;75: 371-7.

[17] Merrill DR, Bikson M, Jefferys JG. Electrical stimulation of excitable tissue: design of efficacious and safe protocols. J Neurosci Methods 2005; 141: 171-98. 
Figure legends

\section{Figure 1: Head phantom and insulated DBS lead for TMS}

The head phantom was filled with gelatin, which was adjusted in thickness and impedance to be similar to those of human brain. The DBS lead was inserted into the phantom through a hole in the left frontal position (A). The tip of lead was positioned at the hypothetical subthalamic nucleus (B). The extra lead was fixed with or without double loops near the insertion point.

\section{Figure 2: Correlation between TMS intensity and size of induced current}

The correlation between size of induced current and TMS intensity showed a linear relationship for each pair of coil position and coil type. The DBS lead was fixed on the model without looping in this situation. (Filled circle indicates the figure 8 coil over the lead, triangle ring coil over the $\mathrm{Cz}$ position, and cubic double cone coil over the $\mathrm{Cz}$ position.) The raw trace of the induced current with $40 \%$ TMS using a figure 8 coil positioned over the lead is shown in the left corner. The area of negative phase was slightly larger than the subsequent long-lasting positive phase (103\%), indicating that this evoked current was not precisely charge balanced.

\section{Figure 3: Relationship between the size of the induced current and the TMS coil position.}

The size of induced current was expressed as peak-to-peak amplitude. The DBS lead was fixed on the surface of scalp type simulation model without looping (A) or after forming double loops as shown in figure 1A (B). The figure 8 coil with $50 \%$ stimulus intensity was systematically moved and the intensity was plotted 3 dimensionally. The maximal amplitude was recorded when the coil was positioned over the lead or over the loop. 
Figure 4: Relationship between charge density and TMS coil position.

The charge density of the first phase of the induced current was calculated and plotted. The coil, stimulus intensity, and recording conditions were the same as those in figure 3 . The induced charge density was maximized when the loops were positioned around the center of the TMS coil. 
Figure. 1

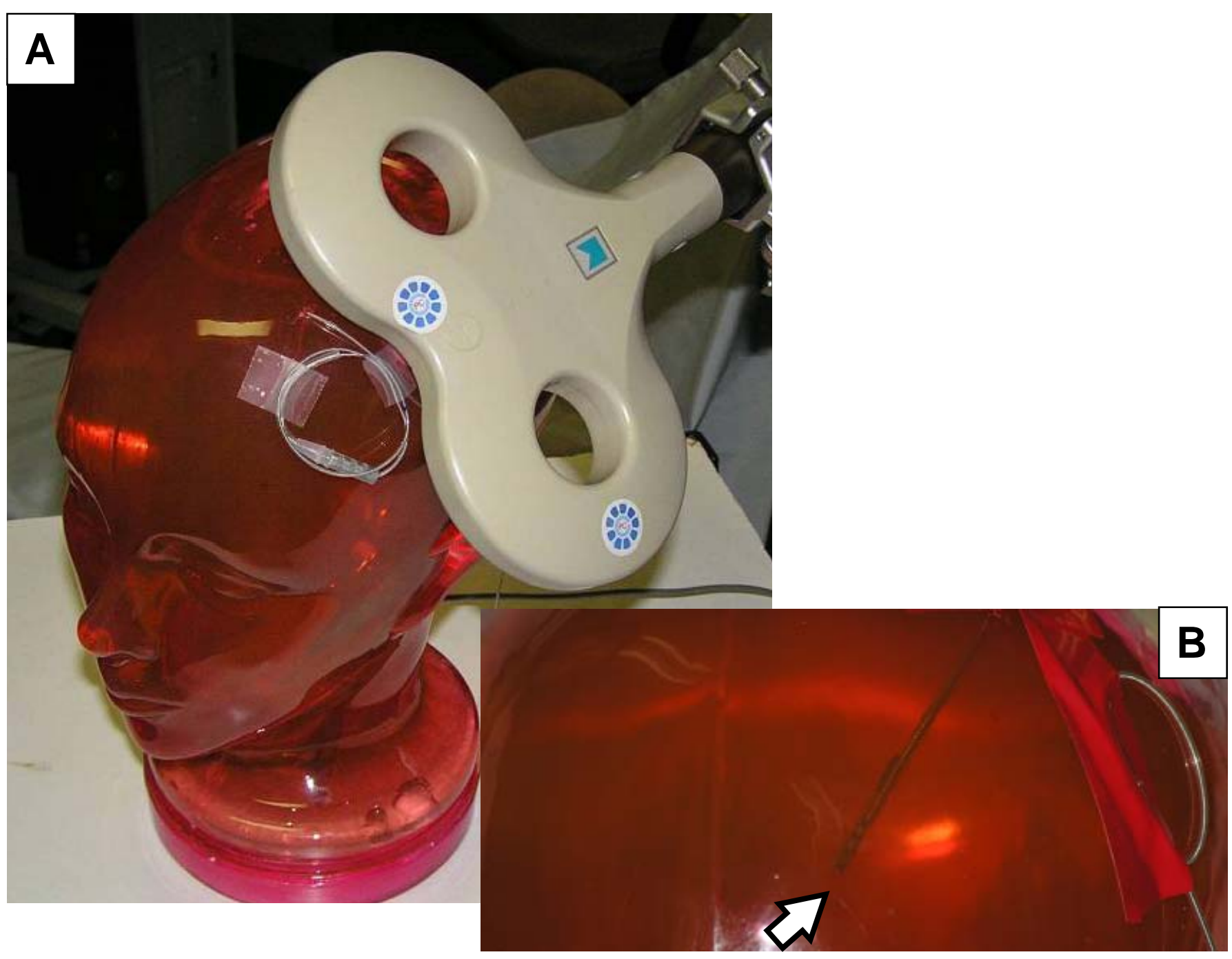


Figure. 2

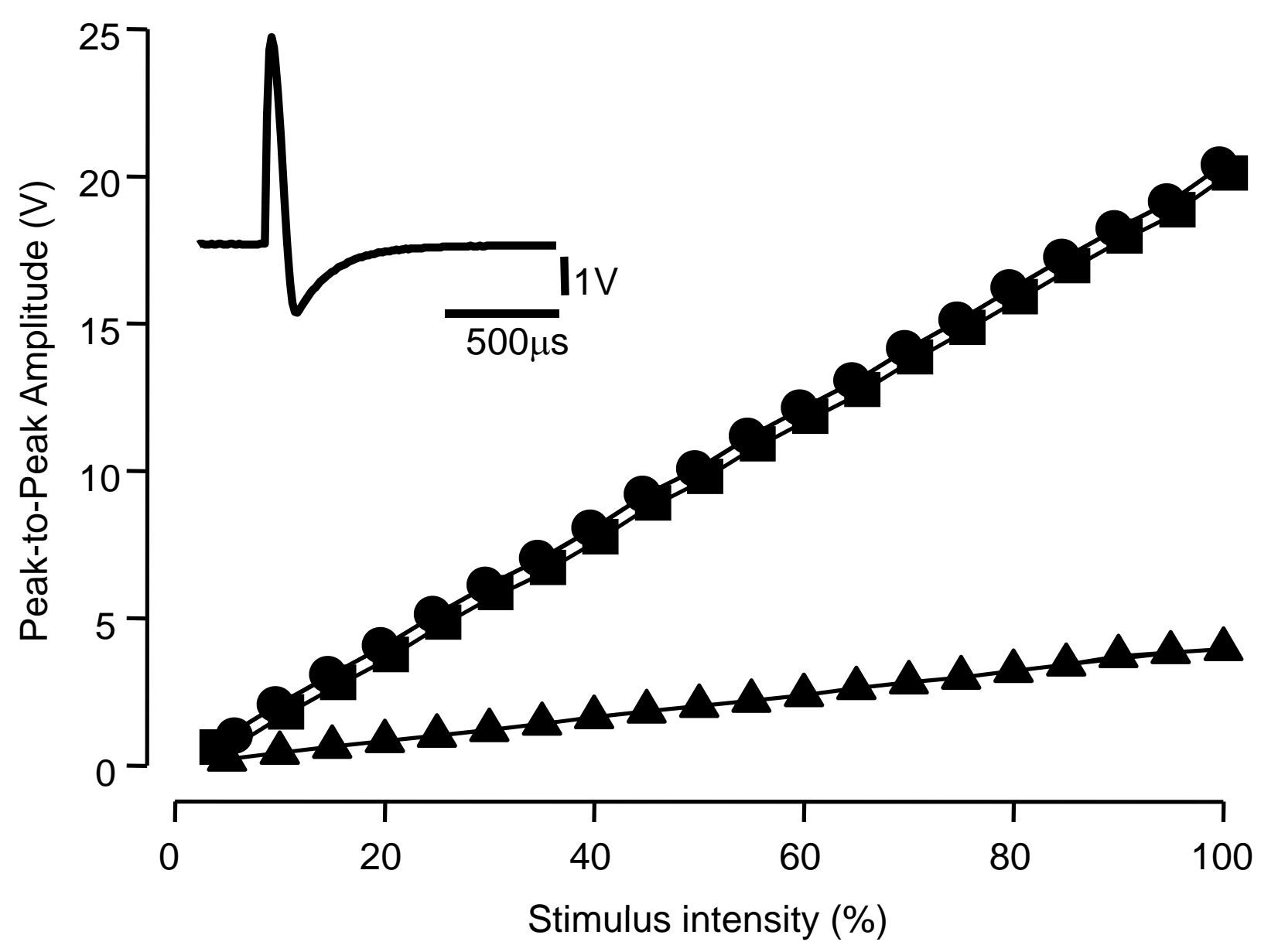


Figure. 3
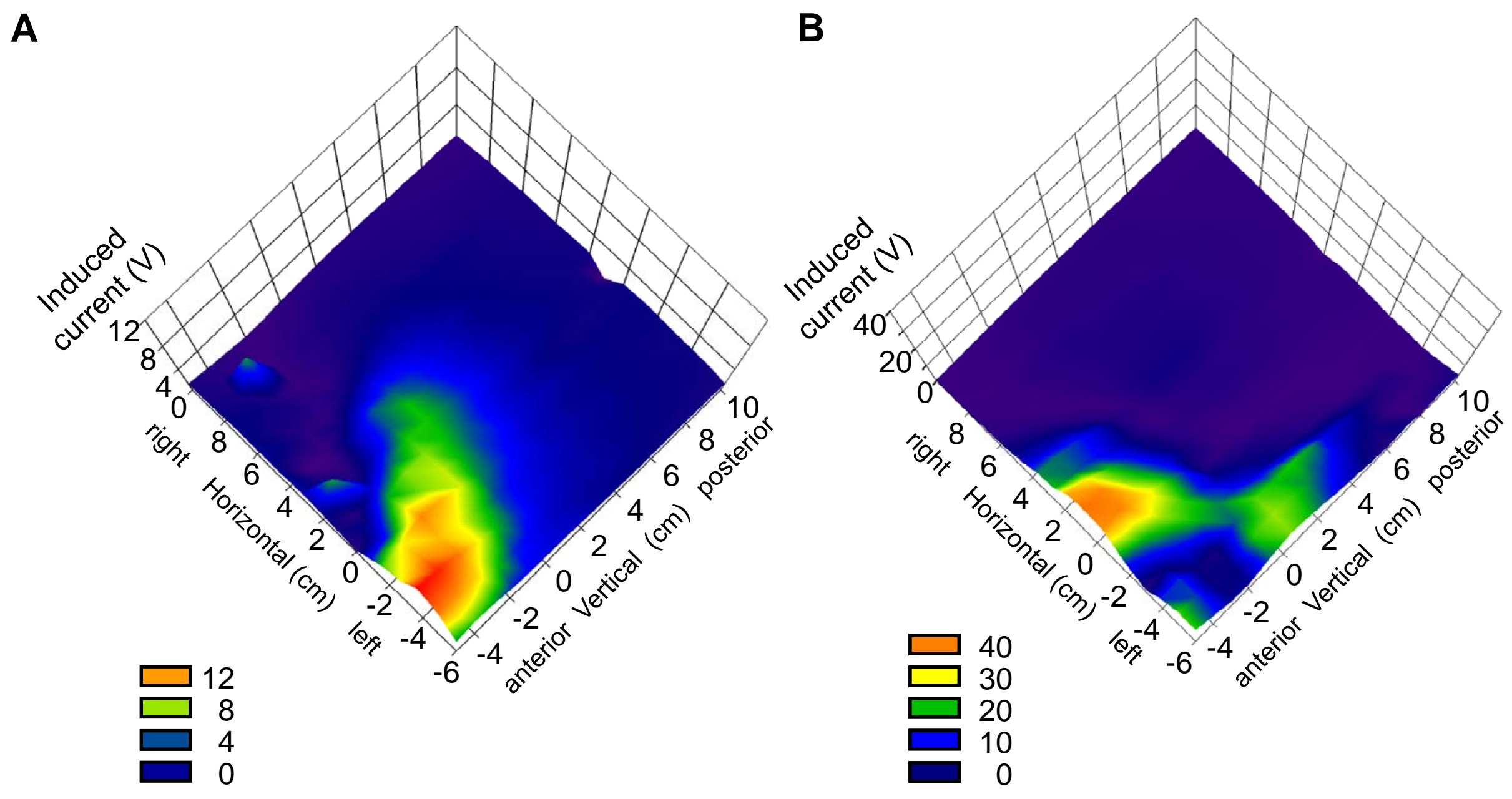
Figure. 4
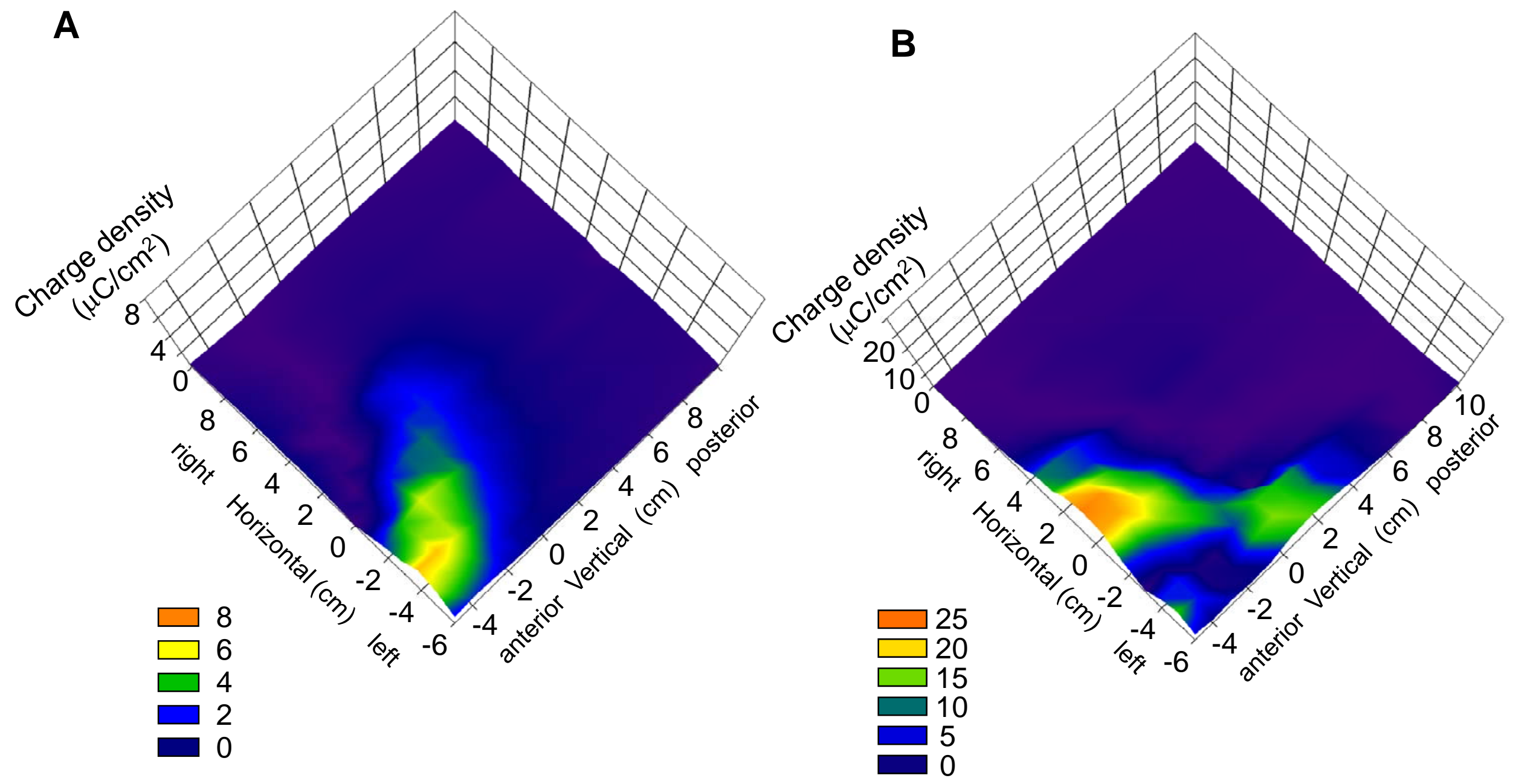